\title{
Magnetic Properties of Some Nickel(II)-Substituted Propanoate Dimers and the Crystal Structure of Tetrakis $(\boldsymbol{\mu}$-2-methyl-2-phenylpropionato- $\left.O, O^{\prime}\right)$ bis(triphenylphosphine)dinickel(II), $\left[\mathrm{Ni}\left(\mathrm{Me}_{2} \mathrm{PhCCOO}\right)_{2} \mathrm{PPh}_{3}\right]_{2}$
}

\author{
Steinar Husebye, ${ }^{\dagger, \mathrm{a}}$ Michinobu Kato, ${ }^{\mathrm{b}}$ Knut Maartmann-Moe, ${ }^{\text {a }}$ Yoneichiro Muto, ${ }^{\mathrm{c}}$ \\ Michio Nakashima ${ }^{\mathrm{c}}$ and Tadashi Tokii ${ }^{\mathrm{c}}$
}

\begin{abstract}
${ }^{a}$ Department of Chemistry, University of Bergen, N-5007 Bergen, Norway, 'bepartment of Chemistry, Aichi Prefectural University, Mizuho-ku, Nagoya, 467 Japan and ${ }^{\mathrm{c}}$ Department of Chemistry, Faculty of Science and Engineering, Saga University, Saga, 840 Japan
\end{abstract}

\begin{abstract}
Husebye. S.. Kato. M.. Maartmann-Moe. K.. Muto. Y.. Nakashima. M. and Tokii, T.. 1994. Magnetic Properties of Some Nickel(II)-Substituted Propanoate Dimers and the Crystal Structure of Tetrakis( $\mu$-2-methyl-2-phenylpropionato$\left.O, O^{\prime}\right)$ bis(triphenylphosphine)dinickel(II), $\left[\mathrm{Ni}\left(\mathrm{Me}_{2} \mathrm{PhCCOO}\right)_{2} \mathrm{PPh}_{3}\right]_{2}$. - Acta Chem. Scand. 48: 628-634 (O) Acta Chemica Scandinavica 1994.

The dimeric $\mathrm{Ni}(\mathrm{II})$ complexes $\left[\mathrm{Ni}(\mathrm{RCOO})_{2} \mathrm{~L}\right]_{2}\left(\mathbf{1}, \mathrm{R}=\mathrm{Me}_{2} \mathrm{PhC}, \mathrm{L}=\mathrm{PPh}_{3} ; \mathbf{2}\right.$, $\mathrm{R}=\mathrm{Me}_{2} \mathrm{PhC}, \mathrm{L}=$ quinoline and $3, \mathrm{R}=\mathrm{Me}_{3} \mathrm{C}, \mathrm{L}=2$-picoline) have been synthesized. They all display a dimer type of antiferromagnetism found in similar $\mathrm{Cu}(\mathrm{II})$ and $\mathrm{Ni}(\mathrm{II})$ complexes. The crystal structure of $\mathbf{1}$ has been determined from X-ray diffraction data at $295 \mathrm{~K}$. The green crystals are triclinic with $a=12.001(5)$, $b=14.688(5), c=19.666(6) \AA, \alpha=86.13(2), \beta=82.57(3), \gamma=76.718(3), \quad Z=2$, space group $P \bar{l}$. Full-matrix least-squares refinement based on 5589 observed reflections gave a final $R=0.085$. There are two crystallographically independent half molecules in the asymmetric unit. The compound has the classical dimeric structure well known for numerous $\mathrm{Cu}(\mathrm{II})$ carboxylates. Thus $\mathrm{Ni}(\mathrm{II})$ has a squarepyramidal five-coordination with $\mathrm{P}$ apical and $\mathrm{Ni}$ on average $0.276 \AA$ above the basal oxygen plane. The $\mathrm{Ni} \cdots \mathrm{Ni}$ separations in the dimers average $2.759 \AA$.
\end{abstract}

Only relatively recently the first $\mathrm{Ni}(\mathrm{II})$ carboxylate dimers analogous to the $\mathrm{Cu}(\mathrm{II})$ carboxylate dimers have been synthesized and characterized by magnetic measurements and X-ray diffraction studies. ${ }^{1-4}$ Magnetic susceptibility measurements of these compounds show an antiferromagnetic coupling, but the nature of the spin interaction has not been established with certainty. It is probably of the same type as that found in the corresponding $\mathrm{Cu}$ (II) dimers. ${ }^{5}$ The present study is part of an effort to find a correlation between structure and magnetism in $\mathrm{Ni}(\mathrm{II})$ carboxylate dimers.

\section{Experimental}

Synthesis. [ $\left.\mathrm{Ni}\left(\mathrm{Me}_{2} \mathrm{PhCCOO}\right)_{2} \mathrm{~L}\right]_{2}\left(\mathrm{~L}=\mathrm{PPh}_{3}, \mathbf{1}\right.$, quin, 2$)$ was prepared as follows. Nickel(II) acetate tetrahydrate (2 mmol), 2-methyl-2-phenylpropanoic acid (4 mmol), and $\mathrm{L}(2 \mathrm{mmol})$ were dissolved in 1-butanol $(30 \mathrm{ml})$ by

\footnotetext{
${ }^{\dagger}$ To whom correspondence should be addressed.
}

heating at ca. $80^{\circ} \mathrm{C}$, and this temperature was kept for $30 \mathrm{~min}$. After filtration the solution was concentrated to ca. $2 \mathrm{ml}$ by evaporation in vacuo with a bath temperature of ca. $80^{\circ} \mathrm{C}$. After adding $20 \mathrm{ml}$ of $p$-xylene the solution was concentrated to ca. $5 \mathrm{ml}$. Petroleum ether $(0.5 \mathrm{ml})$ was added to the solution and green crystals appeared after keeping at room temperature for $1 \mathrm{~h}$. The crystals were collected and washed with a 1:1 mixture of petroleum ether and $p$-xylene, and dried in vacuo at room temperature. Yield: ca. 0.6 g. $\left[\mathrm{Ni}\left(\mathrm{Me}_{3} \mathrm{CCOO}\right)_{2}(2 \text {-pic })\right]_{2}, 3$, was prepared by the same method and reported earlier. ${ }^{6}$ Anal. 1: Found: C, 70.66; H, 5.78; Ni, 9.00\%. Calc. for $\mathrm{C}_{76} \mathrm{H}_{74} \mathrm{Ni}_{2} \mathrm{O}_{8} \mathrm{P}_{2}: \mathrm{C}, 70.50 ; \mathrm{H}, 5.76 ; \mathrm{Ni}, 9.07 \%$. 2: Found: C, 68.07; H, 5.77; N, 2.65; Ni, $11.51 \%$. Calc. for $\mathrm{C}_{58} \mathrm{H}_{58} \mathrm{~N}_{2} \mathrm{Ni}_{2} \mathrm{O}_{8}: \mathrm{C}, 67.72 ; \mathrm{H}, 5.70 ; \mathrm{N}, 2.72 ; \mathrm{Ni}, 11.41 \%$. 3: Found: C, $54.38 ; \mathrm{H}, 7.16 ; \mathrm{N}, 3.94 ; \mathrm{Ni}, 16.50 \%$. Calc. for $\mathrm{C}_{52} \mathrm{H}_{58} \mathrm{~N}_{2} \mathrm{Ni}_{2} \mathrm{O}_{8}: \mathrm{C}, 54.27 ; \mathrm{H}, 7.21 ; \mathrm{N}, 3.94 ; \mathrm{Ni}$, $16.58 \%$.

Structure determination. Some of the relevant data are shown in Table 1. Intensities were collected on an Enraf- 
Table 1. Crystal and experimental data.

\begin{tabular}{|c|c|}
\hline Compound & {$\left[\mathrm{Ni}\left\{\mathrm{Me}_{2}(\mathrm{Ph}) \mathrm{CCOO}\right\}_{2} \mathrm{PPh}_{3}\right]_{2}$} \\
\hline Diffractometer & Enraf-Nonius CAD-4 \\
\hline Radiation & $\operatorname{MoK} \alpha$ \\
\hline Wavelength/Å & 0.70930 \\
\hline Crystal system & Triclinic \\
\hline$a / \AA$ & $12.001(5)$ \\
\hline$b / \AA$ & $14.688(5)$ \\
\hline$c / \AA$ & $19.666(6)$ \\
\hline$\alpha /{ }^{\circ}$ & $86.13(3)$ \\
\hline$\beta /{ }^{\circ}$ & $82.57(3)$ \\
\hline$\gamma /{ }^{\circ}$ & $76.72(3)$ \\
\hline$V / \AA^{3}$ & 3342.9 \\
\hline Space group & $P \overline{1}$ \\
\hline Formula wt. & 1298.80 \\
\hline & 2 \\
\hline Dcalc $/ \mathrm{gcm}^{-3}$ & 1.286 \\
\hline$\mu(\mathrm{Mo} K \alpha) / \mathrm{cm}^{-1}$ & 6.65 \\
\hline Crystal dimensions $/ \mathrm{mm}$ & $0.11 \times 0.29 \times 0.40$ \\
\hline Scan mode $/ \max \theta /^{\circ}$ & Omega/25 \\
\hline Fudge factor, $p$ & 0.020 \\
\hline Scale factor & 2.973 \\
\hline No. independent measurements & 11660 \\
\hline No. with $1>2.5 \sigma(/)$ & 5691 \\
\hline Absorption (max/min) & 0.9283 and 0.8310 \\
\hline$R=\Sigma\left|F_{0}-F_{\mathrm{c}}\right| / \Sigma F_{0}$ & 0.085 \\
\hline$R_{w}=\left[\Sigma w\left(F_{0}-F_{c}\right)^{2} / \Sigma w F_{0}^{2}\right]^{1 / 2}$ & 0.083 \\
\hline$S=\left[\sum w(\Delta F)^{2} /(N-n)\right]^{1 / 2}$ & 2.408 \\
\hline
\end{tabular}

Nonius CAD-4 diffractometer at $295 \mathrm{~K}$. The intensities of three standard reflections were checked every $2 \mathrm{~h}$. Their intensities decreased $17.1 \%$ during intensity collection, and an appropriate scaling factor was applied. The data were also collected for Lorentz and polarization effects and for absorption. Unit-cell parameters are based on least-squares fits to the diffractometer settings of 25 general reflections. The structure was solved by conventional heavy-atom methods and refined by full-matrix least-squares methods minimizing $\Sigma w(\Delta F)$. During refinement, several reflections with low $\theta$-values had relatively large $\Delta F$-values. Refinement of some of the lighter atoms also was difficult. For these reasons, reflections with $\sin \theta / \lambda \leq 0.123$ (102 in all) were excluded from the final refinements. As a result, e.s.d. values of atomic parameters improved.

Because of the large number of parameters, only the $\mathrm{Ni}, \mathrm{P}$ and $\mathrm{O}$ atoms were refined with anisotropic temperature factors. $\mathrm{H}$ atoms were placed in calculated positions $(\mathrm{C}-\mathrm{H}=0.95 \AA$ ) with isotropic temperature factors equal to 1.3 times those of the connected $\mathrm{C}$ atoms. Temperature factors of the phenyl groups, especially in the acid ligands, were high and some bond lengths and angles had unrealistic values, indicating possible disorder in these groups. This effect and the crystal decay is probably responsible for the high final $R$-factor of 0.085 . The final difference map showed no maxima above 0.76 or below $-0.57 \mathrm{e} \AA^{-3}$. The choice of the space group $P 1$ rather than the alternative $P \overline{1}$ was based on $E$-statistics and the result of the refinement of the structure. Computer programs used were supplied by Enraf-Nonius (SPD/VAX V3).

Magnetic measurement. Magnetic susceptibilities were determined in the temperature range $80-300 \mathrm{~K}$ by the Faraday method. The diamagnetic contribution was corrected using Pascal's constants. The results are shown in Fig. 3. The cryomagnetic data were analyzed with eqn. (1), (Fig. 3) which is derived from eqn. (2) by the simplification described below.

$$
\begin{aligned}
\chi_{\mathrm{A}}= & \frac{n(n+2)}{3} \frac{N g^{2} \beta^{2}}{3 k T}\left[1+\frac{1}{3} \exp \left(\frac{-2 J}{k T}\right)\right]^{-1}(1-P) \\
& +\frac{2}{3} \frac{N g_{\text {im }} \beta^{2}}{k T} P+N \alpha \\
\chi_{\mathrm{A}}= & \frac{(n+2)}{3} \sum_{i=1}^{2 n} \frac{N g_{i}^{2} \beta^{2}}{3 k T} \\
& \times\left[1+\frac{1}{3} \exp \left(\frac{-2 J_{i}}{k T}\right)\right]^{-1} P_{i}+N \alpha
\end{aligned}
$$
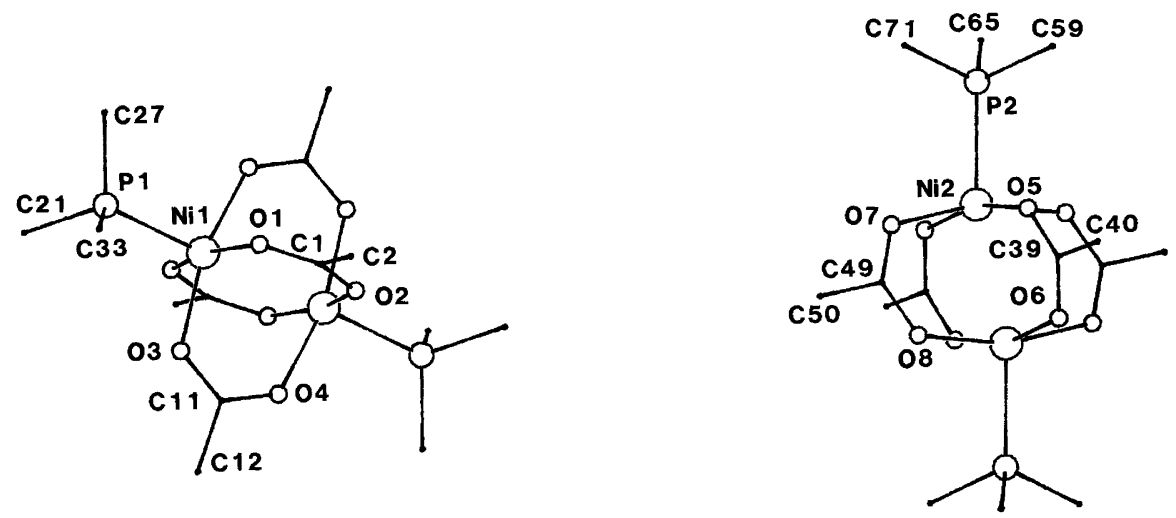

Fig. 1. The structures of the two crystallographically independent and centrosymmetric molecules of the dimer $\left[\mathrm{Ni}\left(\mathrm{Me}_{2} \mathrm{PhCCOO}_{2} \mathrm{PPh}_{3}\right]_{2}\right.$. Methyl and phenyl groups on the carboxylic ligands are omitted, the phenyl groups on $\mathrm{PPh}_{3}$ are indicated by the $\mathrm{C}$ atoms bonded to $\mathrm{P}$. 
HUSEBYE ET AL.

Table 2. Magnetic parameters.

\begin{tabular}{|c|c|c|c|c|c|c|c|c|c|}
\hline \multicolumn{2}{|c|}{ Dimeric $\mathrm{Ni}(\mathrm{II})$ complex } & \multicolumn{5}{|c|}{$\chi-T$ data } & \multicolumn{2}{|l|}{ Crystal data } & \multirow[b]{2}{*}{ Ref. etc. } \\
\hline Carboxylate & $\mathrm{L}^{a}$ & $-J$ & $\mathrm{~g}$ & $N \alpha$ & $P(\%)$ & DI(\%) & Space group & $Z$ & \\
\hline $\mathrm{Me}_{3} \mathrm{CCOO}$ & $2,5-\mathrm{lu}$ & 126 & 2.00 & 400 & 1.72 & 0.67 & $C 2 / c$ & 4 & $(2),(3)$ \\
\hline $\mathrm{Me}_{2} \mathrm{PhCCOO}$ & $\mathrm{PPh}_{3}$ & 206 & 2.00 & 440 & 0.18 & 0.86 & $P \overline{1}$ & 2 & This work \\
\hline $\mathrm{Me}_{2} \mathrm{PhCCOO}$ & quin & 142 & 2.03 & 450 & 1.34 & 1.31 & $P 2_{1} / n$ & 2 & This work (3) \\
\hline $\mathrm{Me}_{3} \mathrm{CCOO}$ & 2-pic & 223 & 2.28 & 320 & 0.07 & 1.38 & $P \underline{1}$ & 1 & This work (3) \\
\hline $\mathrm{Me}_{3} \mathrm{CCOO}$ & 2-Etpy & 224 & 2.44 & 380 & 0.09 & 1.21 & $P \overline{1}$ & 1 & $(2),(3)$ \\
\hline $\mathrm{Me}_{3} \mathrm{CCOO}$ & $2,4-l u$ & 180 & 2.71 & 400 & 0.22 & 0.54 & $P \overline{1}$ & 1 & (2) High-T \\
\hline $\mathrm{Me}_{3} \mathrm{CCOO}$ & $2,4-l u$ & 192 & 2.01 & 310 & 0.04 & 1.12 & - & - & (2) Low-T \\
\hline
\end{tabular}

${ }^{a}$ lu, lutidine; quin, quinoline; pic, picoline; Etpy, ethylpyridine.

Table 3. Positional parameters and their estimated standard deviations.

\begin{tabular}{|c|c|c|c|c|c|c|c|c|c|}
\hline Atom & $x$ & $y$ & $z$ & $B / \AA^{2}$ & Atom & $x$ & $y$ & $z$ & $B / \AA^{2}$ \\
\hline $\mathrm{Ni} 1$ & $0.08004(9)$ & $0.04021(8)$ & $0.02332(6)$ & $29(2)$ & C33 & $0.1414(7)$ & $0.2528(6)$ & $0.0639(5)$ & 3. $1(2)^{*}$ \\
\hline $\mathrm{Ni} 2$ & $0.3891(1)$ & $0.51036(9)$ & $0.53295(6)$ & $2.85(3)$ & C34 & $0.1856(9)$ & $0.3239(8)$ & $0.0290(6)$ & $5.2(3)^{*}$ \\
\hline P1 & $0.2060(2)$ & $0.1288(2)$ & $0.0569(1)$ & $2.58(5)$ & C35 & $0.133(1)$ & $0.4171(8)$ & $0.0421(6)$ & $6.4(3)^{*}$ \\
\hline P2 & $0.2200(2)$ & $0.5187(2)$ & $0.6132(1)$ & $3.62(6)$ & C36 & $0.035(1)$ & $0.4390(8)$ & $0.0858(6)$ & $5.8(3)^{*}$ \\
\hline 01 & $0.0073(5)$ & $0.1472(4)$ & $-0.0365(3)$ & $3.7(2)$ & C37 & $-0.010(1)$ & $0.3718(8)$ & $0.1204(6)$ & $5.7(3)^{*}$ \\
\hline $\mathrm{O} 2$ & $-0.1244(5)$ & $0.0844(4)$ & $-0.0722(3)$ & $3.9(2)$ & C38 & $0.0426(9)$ & $0.2773(7)$ & $0.1096(5)$ & $4.5(2)^{*}$ \\
\hline $\mathrm{O} 3$ & $-0.0453(5)$ & $0.0811(4)$ & $0.1002(3)$ & $3.7(2)$ & C39 & $0.5802(8)$ & $0.3578(6)$ & $.5769(5)$ & $3.2(2)^{*}$ \\
\hline 04 & $-0.1734(5)$ & $0.0189(4)$ & $0.0610(3)$ & $3.9(2)$ & $\mathrm{C} 40$ & $0.6211(9)$ & $0.2756(7)$ & $0.6256(5)$ & $4.4(2)^{*}$ \\
\hline 05 & $0.4758(5)$ & $0.3962(5)$ & $0.5822(3)$ & $4.2(2)$ & C41 & $0.520(1)$ & $0.256(1)$ & $0.6758(8)$ & $9.4(4)^{*}$ \\
\hline 06 & $0.6575(5)$ & $0.3810(5)$ & $0.5348(3)$ & $4.5(2)$ & C42 & $0.699(2)$ & $0.305(1)$ & $0.668(1)$ & $12.2(5)^{*}$ \\
\hline 07 & 0.36 & 0.4 & 0.4 & & $\mathrm{C} 43$ & & & (8) & $1(4)^{*}$ \\
\hline 08 & 0.5 & 0.4 & & $3.7(2)$ & $\mathrm{C} 44$ & & & & $14.3(6)^{*}$ \\
\hline C1 & -0.07 & 0.1 & -0.0 & $3.2(2)^{*}$ & C45 & 0.8 & 0.0 & 0.5 & $17.7(8)^{*}$ \\
\hline $\mathrm{C} 2$ & $-0.1225(8)$ & $0.2443(7)$ & $-0.1088(5)$ & $3.8(2)^{*}$ & $\mathrm{C} 46$ & & & & $16.0(7)^{*}$ \\
\hline C3 & $-0.156(1)$ & 0.22 & $-0.1773(6)$ & & C47 & & & & $(9)^{*}$ \\
\hline C4 & $-0 . c$ & 0.2 & -0 & & C48 & & & & $(6)^{*}$ \\
\hline C5 & -0.2 & & & 4.2 & C49 & & & & $3.6(2)^{*}$ \\
\hline C6 & -0.3 & 0.3 & $-0.0792(7)$ & $7.9(4)^{*}$ & $\mathrm{C} 50$ & $2(8)$ & $0.3117(7)$ & 0.3 & $3.9(2)^{*}$ \\
\hline C7 & $-0.421(1)$ & $0.371(1)$ & -0.0 & $11.1(5)^{*}$ & C51 & & $0.2603(8)$ & (6) & $5.6(3)^{*}$ \\
\hline C8 & -0.3 & & & & & & & & $(3)^{*}$ \\
\hline C9 & -0.2 & & & & $\mathrm{C5}$ & & & & $4.3(2)^{*}$ \\
\hline C10 & -0.1 & 0.3 & $-0 . c$ & $7.8(4)^{*}$ & C54 & & & (8) & $9.3(4)^{*}$ \\
\hline C11 & $-0.1439(7)$ & $0.0639(6)$ & $0.1034(4)$ & $3.0(2)^{*}$ & C55 & & & & $13.5(6)^{*}$ \\
\hline C12 & $-0.2302(8)$ & & & & & & & & \\
\hline $\mathrm{C} 13$ & -0.2 & 0.1 & & & & & & & \\
\hline C14 & -0.3 & 0.1 & & 3)* & C & & & & $7.6(3)^{*}$ \\
\hline C15 & $-0.231(1)$ & 0.02 & $3(6)$ & $6.9(3)^{*}$ & C59 & $6(8)$ & $0.4743(7)$ & & $3.9(2)^{*}$ \\
\hline C16 & $-0.141(2)$ & $-0.022(1)$ & & $13.2(6)^{*}$ & $\mathrm{C} 60$ & & $0.5099(8)$ & & $5.7(3)^{*}$ \\
\hline$C_{17}$ & -0 & 0 & & & & & & & $(3)^{*}$ \\
\hline C18 & -0.2 & -0 & & & $\mathrm{CC}$ & & 8) & & $6.6(3)^{*}$ \\
\hline C19 & -0.3 & -0.0 & 0. & & C63 & & $0.3766(9)$ & $4(7)$ & $7.4(3)^{*}$ \\
\hline $\mathrm{C} 2 \mathrm{O}$ & $-0.340(2)$ & $0.009(1)$ & $0.2450(9)$ & $11.7(5)^{*}$ & C64 & & $0.4063(8)$ & & $6.4(3)^{*}$ \\
\hline $\mathrm{C} 21$ & & & & & & & & & $4.5(2)^{*}$ \\
\hline $\mathrm{C} 22$ & & & & & & & & & \\
\hline $\mathrm{C} 23$ & 0 & -0 & 0. & 3)* & CE & $0 . c$ & 0.7 & 0.6 & $8.3(4)^{*}$ \\
\hline C24 & $0.3132(9)$ & $0.0516(7)$ & $0.2723(5)$ & $4.9(2)^{*}$ & C68 & $-0.008(1)$ & $0.809(1)$ & $0.6573(7)$ & $8.4(4)^{*}$ \\
\hline C25 & $0.3369(9)$ & $0.1279(7)$ & $0.2398(5)$ & $4.5(2)^{*}$ & C69 & $-0.025(1)$ & $0.7364(9)$ & $0.6944(7)$ & $7.8(4)^{*}$ \\
\hline C26 & $0.3056(8)$ & $0.1554(7)$ & & & C7O & & & & $6.9(3)^{*}$ \\
\hline $\mathrm{C} 27$ & & & -0.0 & & C71 & & $0.4464(7)$ & & $3.9(2)^{*}$ \\
\hline C28 & & 019 & 0 & 4.7 & C7 & & $2(8)$ & & $5.6(3)^{*}$ \\
\hline C29 & 0 & $0.1214(8)$ & $-0.0372\langle 6\rangle$ & $5.9(3)^{*}$ & C73 & $0.112(1)$ & $0.2963(9)$ & $0.5518(7)$ & $7.6(3)^{*}$ \\
\hline C30 & $0.514(1)$ & $0.1371(8)$ & $-0.1032(6)$ & $5.8(3)^{*}$ & C74 & -0.0 & $0.3341(9)$ & $0.5513(7)$ & $7.5(3)^{*}$ \\
\hline C31 & $0.407(1)$ & $0.1469(8)$ & -0.12 & $6.4(3)^{*}$ & $\mathrm{C} 7 \mathrm{~S}$ & -0.0 & & $0.5677(6)$ & $6.4(3)^{*}$ \\
\hline 0 & $0.3156(9)$ & $0.1406(7)$ & $-0.0729(5)$ & $4.9(2)^{*}$ & C76 & $0.007(1)$ & $0.4802(8)$ & $0.5880(6)$ & $5.8(3)^{*}$ \\
\hline
\end{tabular}

Starred atoms were refined isotropically. Anisotropically refined atoms are given in the form of the isotropic equivalent displacement parameter defined as: $(4 / 3)^{*}\left[a^{2 *} B(1,1)+b^{2 *} B(2,2)+c^{2 *} B(3,3)+a b(\cos \gamma)^{*} B(1,2)+a c(\cos \beta)^{*} B(1,3)+\right.$ $\left.b c(\cos \alpha)^{*} B(2,3)\right]$. 
Eqn. (2) is a modified expression of a channel model developed by Rakitin et al., ${ }^{7}$ where $n$ is the number of unpaired electrons on one metal ion $\left(n=2\right.$ for the $\mathrm{Ni}^{2+}$ $\mathrm{Ni}^{2+}$ dimer), $P_{i}$ is the mole fraction of each component, and the other symbols have their usual meanings. For the unpaired electrons in the dimeric molecule $i=1, \cdots n$, and for the electrons in the monomeric impurity $i=n+1, \cdots 2 n$ and $J_{n+1}, \cdots J_{2 n}=0$. When the values of $g\left(g_{1}=g_{2}=g\right)$ and $J\left(J_{1}=J_{2}=J\right)$ for the dimeric unit and those of $g_{\text {im }}\left(g_{3}=g_{4}=g_{\text {im }}\right), J_{3}=J_{4}=0$, and $P\left(P_{3}=P_{4}=P\right)$ for the paramagnetic impurity are used, eqn. (1) is obtained. The best-fit parameters for $g, J, P$ and $N \alpha$ were obtained by a nonlinear least-squares fitting procedure assuming $g_{\text {im }}=2.2$. The quality of fit was estimated by means of the discrepancy index, $\sigma_{\text {dis }}=\left[\Sigma\left(\chi_{\text {obs }}-\chi_{\text {calc }}\right)^{2} / \Sigma \chi_{\text {obs }}^{2}\right]^{1 / 2}$. The values of the magnetic parameters are summarized in Table 2 together with some crystallographic data.

\section{Results and discussion}

Magnetic parameters are listed in Table 2, atomic positional parameters in Table 3, bond distances and angles in Tables 4 and 5 and molecular planes in Table 6. Further details of the crystal structure work are deposited with the Cambridge Crystallographic Data Centre, UK.

Molecular structure. The structures of the two crystallographically independent and centrosymmetric molecules $\mathbf{1 a}$ and $\mathbf{1 b}$ are shown in Fig. 2. They are quite similar. The carboxylate ligands are all bridging and the dimers have the cage structure well known from the $\mathrm{Cu}$ (II) carboxylate dimers with a somewhat distorted square-pyramidal coordination of the metal atom. The $\mathrm{PPh}_{3}$ group is apical, while four oxygen atoms form the basal plane. The main difference between the two molecules is represented by the $\mathrm{Ni}^{\prime}-\mathrm{Ni}-\mathrm{P}$ angles, which are $172.43(7)$ and $166.65(6)^{\circ}$ for $\mathbf{1 a}$ and $\mathbf{1 b}$. They agree with those of other dimeric $\mathrm{Ni}(\mathrm{II})$ carboxylates, but the angle in $\mathbf{1 a}$ is a bit high. ${ }^{1-3}$ Figure 3 shows the packing of molecules in the unit cell. No short intermolecular distances were found.

Bonding. The Ni-Ni distances for $\mathbf{1 a}$ and $\mathbf{1 b}$ are 2.752(1) and $2.765(2) \AA$, respectively, and may be compared to distances in the range $2.708-2.765$ found earlier for such compounds. ${ }^{1-3}$ The distances are too large for a $\mathrm{Ni}-\mathrm{Ni}$

Table 4. Bond lengths with estimated standard deviations (in $\AA$ ).

\begin{tabular}{|c|c|c|c|c|c|}
\hline $\mathrm{Ni} 1-\mathrm{Ni} 1^{\prime a}$ & $2.752(1)$ & $\mathrm{C} 8-\mathrm{C} 9$ & $1.37(1)$ & C43-C44 & $1.46(2)$ \\
\hline aNi1-P1 & $2.386(2)$ & $\mathrm{C} 9-\mathrm{C} 10$ & $1.41(1)$ & C43-C48 & $1.28(2)$ \\
\hline $\mathrm{Ni} 1-\mathrm{O} 1$ & $2.001(4)$ & C11-C 12 & $1.514(8)$ & C44-C45 & $1.56(2)$ \\
\hline $\mathrm{Ni} 1-\mathrm{O} 2^{\prime}$ & $2.001(4)$ & C 12-C 13 & $1.501(9)$ & $C 45-C 46$ & $1.25(2)$ \\
\hline $\mathrm{Ni} 1-\mathrm{O} 3$ & $2.008(4)$ & C 12-C14 & $1.56(1)$ & C46-C47 & $1.28(2)$ \\
\hline $\mathrm{Ni} 1-\mathrm{O} 4^{\prime}$ & $2.006(4)$ & C 12-C 15 & $1.45(1)$ & C47-C48 & $1.69(2)$ \\
\hline $\mathrm{Ni} 2-\mathrm{Ni} 2^{\prime}$ & $2.765(2)$ & C $15-C 16$ & $1.26(1)$ & C49-C50 & $1.542(9)$ \\
\hline $\mathrm{Ni2}-\mathrm{P} 2$ & $2.389(2)$ & C 15-C20 & $1.42(1)$ & C50-C5 1 & $1.540(9)$ \\
\hline $\mathrm{Ni2}-\mathrm{O} 5$ & $2.017(4)$ & C $16-C 17$ & $1.67(2)$ & C50-C52 & $1.50(1)$ \\
\hline $\mathrm{Ni} 2-\mathrm{O}^{\prime}$ & $2.032(4)$ & C17-C18 & $1.26(2)$ & C50-C53 & $1.512(9)$ \\
\hline $\mathrm{Ni2}-\mathrm{O} 7$ & $2.007(4)$ & C 18-C 19 & $1.32(2)$ & C53-C54 & $1.29(1)$ \\
\hline $\mathrm{Ni} 2-08^{\prime}$ & $2.014(4)$ & C 19-C20 & $1.47(2)$ & C53-C58 & $1.43(1)$ \\
\hline P1-C21 & $1.802(6)$ & C21-C22 & $1.376(8)$ & C54-C55 & $1.47(2)$ \\
\hline P $1-C 27$ & $1.813(6)$ & $\mathrm{C} 21-\mathrm{C} 26$ & $1.388(8)$ & C55-C56 & $1.27(1)$ \\
\hline P1-C33 & $1.815(6)$ & C22-C23 & $1.406(9)$ & C56-C57 & $1.33(1)$ \\
\hline P2-C59 & $1.822(7)$ & C23-C24 & $1.357(9)$ & C57-C58 & $1.46(1)$ \\
\hline P2-C65 & $1.800(7)$ & C24-C25 & $1.322(9)$ & C59-C60 & $1.372(9)$ \\
\hline P2-C71 & $1.832(7)$ & $C 25-C 26$ & $1.414(9)$ & C59-C64 & $1.366(9)$ \\
\hline $\mathrm{O} 1-\mathrm{C} 1$ & $1.262(7)$ & C27-C28 & $1.348(8)$ & C60-C61 & $1.40(1)$ \\
\hline $\mathrm{O} 2-\mathrm{C} 1$ & $1.243(7)$ & C27-C32 & $1.409(9)$ & C61-C62 & $1.34(1)$ \\
\hline O3-C11 & $1.259(7)$ & C28-C29 & $1.416(9)$ & C62-C63 & $1.33(1)$ \\
\hline O4-C11 & $1.232(7)$ & C29-C30 & $1.35(1)$ & C63-C64 & $1.45(1)$ \\
\hline O5-C39 & $1.245(7)$ & C30-C31 & $1.37(1)$ & C65-C66 & $1.355(9)$ \\
\hline O6-C39 & $1.253(7)$ & C31-C32 & $1.391(9)$ & $\mathrm{C} 65-\mathrm{C} 70$ & $1.40(1)$ \\
\hline O7-C49 & $1.231(7)$ & C33-C34 & $1.381(8)$ & C66-C67 & $1.38(1)$ \\
\hline O8-C49 & $1.268(7)$ & C33-C38 & $1.384(8)$ & C67-C68 & $1.40(1)$ \\
\hline $\mathrm{C} 1-\mathrm{C} 2$ & $1.548(8)$ & C34-C35 & $1.40(1)$ & C68-C69 & $1.29(1)$ \\
\hline $\mathrm{C} 2-\mathrm{C} 3$ & $1.539(9)$ & C35-C36 & $1.34(1)$ & $\mathrm{C} 69-\mathrm{C} 70$ & $1.40(1)$ \\
\hline $\mathrm{C} 2-\mathrm{C} 4$ & $1.548(9)$ & C36-C37 & $1.34(1)$ & C71-C72 & $1.364(9)$ \\
\hline C2-C5 & $1.470(9)$ & C37-C38 & $1.404(9)$ & C71-C76 & $1.404(9)$ \\
\hline C5-C 10 & $1.38(1)$ & C39-C40 & $1.519(9)$ & $C 72-C 73$ & $1.40(1)$ \\
\hline C5-C6 & $1.37(1)$ & C40-C41 & $1.53(1)$ & C73-C74 & $1.38(1)$ \\
\hline C6-C7 & $1.45(1)$ & $\mathrm{C} 40-\mathrm{C} 42$ & $1.49(1)$ & C74-C75 & $1.30(1)$ \\
\hline C7-C8 & $1.28(1)$ & C40-C43 & $1.52(1)$ & C75-C76 & $1.41(1)$ \\
\hline
\end{tabular}

${ }^{a}$ Atoms marked with a prime are related to those of Table 3 by molecular symmetry centres. 
Table 5. Bond angles with estimated standard deviations $/{ }^{\circ}$.

\begin{tabular}{|c|c|c|c|c|c|c|c|}
\hline $\mathrm{Ni} 1^{\prime}-\mathrm{Ni} 1-\mathrm{O} 1^{a}$ & $81.3(1)$ & $\mathrm{Ni} 1-\mathrm{O} 1-\mathrm{C} 1$ & $126.8(4)$ & $\mathrm{P} 1-\mathrm{C} 21-\mathrm{C} 22$ & $119.3(5)$ & O7-C49-C50 & $120.3(7)$ \\
\hline $\mathrm{Ni} 1^{\prime}-\mathrm{Ni} 1-\mathrm{O} 2^{\prime}$ & $83.1(1)$ & $\mathrm{Ni} 1^{\prime}-\mathrm{O} 2-\mathrm{C} 1$ & $125.0(4)$ & $P 1-C 21-C 26$ & $122.4(5)$ & O8-C49-C50 & $114.7(6)$ \\
\hline $\mathrm{Ni} 1^{\prime}-\mathrm{Ni} 1-\mathrm{O} 3$ & $84.0(1)$ & $\mathrm{Ni} 1-\mathrm{O} 3-\mathrm{C} 11$ & $123.1(4)$ & $\mathrm{C} 22-\mathrm{C} 21-\mathrm{C} 26$ & $118.1(6)$ & C49-C50-C51 & $107.8(6)$ \\
\hline $\mathrm{Ni} 1^{\prime}-\mathrm{Ni} 1-\mathrm{O}^{\prime}$ & $80.2(1)$ & $\mathrm{Ni} 1^{\prime}-\mathrm{O} 4-\mathrm{C} 11$ & $128.6(4)$ & $\mathrm{C} 21-\mathrm{C} 22-\mathrm{C} 23$ & $121.4(6)$ & C49-C5O-C52 & $110.1(6)$ \\
\hline $\mathrm{Ni} 1^{\prime}-\mathrm{Ni} 1-\mathrm{P} 1$ & $172.43(7)$ & $\mathrm{Ni2}-05-\mathrm{C} 39$ & $131.1(4)$ & $\mathrm{C} 22-\mathrm{C} 23-\mathrm{C} 24$ & $118.9(6)$ & C49-C5O-C53 & $107.2(5)$ \\
\hline $\mathrm{P} 1-\mathrm{Ni} 1-\mathrm{O} 1$ & $91.3(1)$ & $\mathrm{Ni2}{ }^{\prime}-06-\mathrm{C} 39$ & $118.3(4)$ & $\mathrm{C} 23-\mathrm{C} 24-\mathrm{C} 25$ & $121.1(8)$ & C51-C50-C52 & $110.2(6)$ \\
\hline $\mathrm{P} 1-\mathrm{Ni} 1-\mathrm{O} 2^{\prime}$ & $104.4(1)$ & $\mathrm{Ni2}-\mathrm{O} 7-\mathrm{C} 49$ & $120.2(4)$ & $\mathrm{C} 24-\mathrm{C} 25-\mathrm{C} 26$ & $121.5(6)$ & C51-C50-C53 & $108.8(6)$ \\
\hline $\mathrm{P} 1-\mathrm{Ni1}-\mathrm{O} 3$ & $94.9(1)$ & $\mathrm{Ni} 2^{\prime}-08-\mathrm{C} 49$ & $130.1(4)$ & $\mathrm{C} 21-\mathrm{C} 26-\mathrm{C} 25$ & $118.9(7)$ & C52-C50-C53 & $112.5(6)$ \\
\hline $1-\mathrm{Ni} 1-\mathrm{O}^{\prime}$ & $101.0(1)$ & $\mathrm{O} 1-\mathrm{C} 1-\mathrm{O} 2$ & $123.8(6)$ & $\mathrm{C} 28-\mathrm{C} 27-\mathrm{C} 32$ & $119.6(6)$ & C50-C53-C54 & $5(8)$ \\
\hline $\mathrm{O} 1-\mathrm{Ni} 1-\mathrm{O} 2^{\prime}$ & $164.3(2)$ & $\mathrm{O} 2-\mathrm{C} 1-\mathrm{C} 2$ & $3(6)$ & $1-C 27-C 28$ & (5) & C58 & $.7(7)$ \\
\hline $\mathrm{O} 1-\mathrm{Ni} 1-\mathrm{O} 3$ & $90.2(2)$ & $\mathrm{O} 1-\mathrm{C} 1-\mathrm{C} 2$ & $(6)$ & $\mathrm{P} 1-\mathrm{C} 27-\mathrm{C} 32$ & (5) & 58 & $8(8)$ \\
\hline $\mathrm{O} 1-\mathrm{Ni} 1-\mathrm{O} 4^{\prime}$ & $88.6(2)$ & $2-$ & 5) & $\mathrm{C} 28$ & & 55 & \\
\hline $\mathrm{O} 2{ }^{\prime}-\mathrm{Ni} 1-\mathrm{O} 3$ & $89.6(2)$ & C2-C4 & 10 & C29-C30 & (8) & 56 & \\
\hline $\mathrm{O} 2^{\prime}-\mathrm{Ni} 1-\mathrm{O}^{\prime}$ & $87.3(2)$ & $-\mathrm{C} 2-\mathrm{C} 5$ & $107.0(5)$ & $\mathrm{C} 29-\mathrm{C} 30-\mathrm{C} 31$ & $123.2(8)$ & $-\mathrm{C} 57$ & (1) \\
\hline $\mathrm{O} 3-\mathrm{Ni} 1-\mathrm{O}^{\prime}$ & $164.1(2)$ & $\mathrm{C} 3-\mathrm{C} 2-\mathrm{C} 4$ & $107.6(6)$ & C30-C31-C32 & $118.0(8)$ & $-C 58$ & $125(1)$ \\
\hline $\mathrm{Ni} 2^{\prime}-\mathrm{Ni} 2-\mathrm{O} 5$ & $77.4(2)$ & $\mathrm{C} 3-\mathrm{C} 2-\mathrm{C} 5$ & $113.8(6)$ & C27-C32-C31 & $120.3(7)$ & C53-C58-C57 & $114.5(8)$ \\
\hline $\mathrm{i} 2^{\prime}-\mathrm{Ni} 2-06^{\prime}$ & $87.0(1)$ & $\mathrm{C} 4-\mathrm{C} 2-\mathrm{C} 5$ & $111.4(6)$ & C34-C33-C38 & $118.0(6)$ & & $117.0(6)$ \\
\hline $\mathrm{i} 2^{\prime}-\mathrm{Ni2}-\mathrm{O} 7$ & $86.5(1)$ & $\mathrm{C} 2-\mathrm{C} 5-\mathrm{C} 10$ & $118.8(7)$ & $\mathrm{P} 1-\mathrm{C} 33-\mathrm{C} 34$ & (5) & P2- & $4(5)$ \\
\hline $\mathrm{Ni} 2^{\prime}-\mathrm{Ni} 2-\mathrm{O} 8^{\prime}$ & $77.8(1)$ & $\mathrm{C} 2-\mathrm{C} 5-\mathrm{C} 6$ & $123.4(7)$ & P1-C33-C38 & $117.0(5)$ & 64 & $.6(7)$ \\
\hline i2' $-\mathrm{Ni2}-\mathrm{P} 2$ & $166.65(6)$ & $\mathrm{C} 6-\mathrm{C} 5-\mathrm{C} 10$ & 8) & $34-C 35$ & 7) & 61 & 7(7) \\
\hline 2-Ni2-O5 & $92.3(1)$ & $\mathrm{C} 5-\mathrm{C} 6-\mathrm{C} 7$ & (9) & $-\mathrm{C} 35-\mathrm{C} 36$ & 7) & 562 & $5(8)$ \\
\hline $\mathrm{P} 2-\mathrm{Ni2}-\mathrm{O}^{\prime}$ & $3.6(1)$ & $\mathrm{C} 6-\mathrm{C} 7-\mathrm{C} 8$ & & $-\mathrm{C} 36-\mathrm{C} 37$ & & 63 & (8) \\
\hline $\mathrm{P} 2-\mathrm{Ni} 2-\mathrm{O} 7$ & 10 & 8 & & 38 & & 64 & (9) \\
\hline $\mathrm{P} 2-\mathrm{Ni2}-08^{\prime}$ & $94.1(1)$ & c9- & & 37 & & 63 & $2(8)$ \\
\hline $\mathrm{O} 5-\mathrm{Ni} 2-\mathrm{O}^{\prime}$ & $164.1(2)$ & $c 10$ & 12 & $9-06$ & & & $4(6)$ \\
\hline O5-Ni2-O7 & $87.2(2)$ & $\mathrm{O} 3-\mathrm{C} 11-\mathrm{O} 4$ & & O5-C39- & & $-C 70$ & $120.7(6)$ \\
\hline $\mathrm{O} 5-\mathrm{Ni} 2-08^{\prime}$ & $90.9(2)$ & O3-C11-C12 & $117.2(6)$ & O6-C39-C40 & (6) & P2-C71- & $117.9(6)$ \\
\hline $\mathrm{O} 6^{\prime}-\mathrm{Ni} 2-\mathrm{O} 7$ & $88.7(2)$ & $\mathrm{O} 4-\mathrm{C}_{11}-\mathrm{C} 12$ & $118.6(6)$ & C39-C40-C41 & $3(7)$ & P2-C & $122.9(6)$ \\
\hline $\mathrm{O} 6^{\prime}-\mathrm{Ni} 2-08^{\prime}$ & $88.9(2)$ & $C_{11}-C_{12}-C_{13}$ & $112.4(6)$ & C39-C40-C42 & $106.9(7)$ & C66-C65-C70 & $117.8(7)$ \\
\hline $\mathrm{O} 7-\mathrm{Ni2}-\mathrm{O} 8^{\prime}$ & $164.2(2)$ & C11-C12-C14 & $106.6(6)$ & C39-C40-C43 & $112.2(7)$ & C65-C66-C67 & $123.0(8)$ \\
\hline li $1-P 1-C 21$ & $15.7(2)$ & C11-C12-C15 & $108.6(6)$ & $\mathrm{C} 41-\mathrm{C} 40-\mathrm{C} 42$ & $7(8)$ & $-C 68$ & $8(9)$ \\
\hline i1-P1-C27 & $4.0(2)$ & $C_{13}-C_{12}-C_{14}$ & $107.4(6)$ & C41-C40-C43 & (7) & $-C 69$ & $120(1)$ \\
\hline Vi1-P1-C33 & $113.7(2)$ & $C_{13}-C_{12}-C_{15}$ & 11 & $\mathrm{C} 42-\mathrm{C} 40-\mathrm{C} 43$ & (9) & $-C 70$ & \\
\hline C21-P1-C27 & $107.3(3)$ & C14-C12-C15 & 110 & $\mathrm{C} 40-\mathrm{C} 43-\mathrm{C} 44$ & $117(1)$ & C65- & $117.7(8)$ \\
\hline $\mathrm{C} 21-\mathrm{P} 1-\mathrm{C} 33$ & $101.5(3)$ & C12-C15-C16 & & & & $\mathrm{C} 72-\mathrm{C} 71-\mathrm{C} 76$ & $119.1(7)$ \\
\hline C27-P1-C33 & $103.2(3)$ & C12-C15-C20 & & C44-C43-C48 & & $72-C 73$ & $122.5(8)$ \\
\hline $\mathrm{Ni2}-\mathrm{P} 2-\mathrm{C} 59$ & $112.3(2)$ & C 16-C15-C20 & $119(1)$ & C43-C44-C45 & & C72-C73-C74 & $115.2(9)$ \\
\hline $\mathrm{Ni2}-\mathrm{P} 2-\mathrm{C} 65$ & $117.2(2)$ & $\mathrm{C} 15-\mathrm{C} 16-\mathrm{C} 17$ & $124(1)$ & C44-C45-C46 & $110(2)$ & C73-C74-C75 & $125.1(9)$ \\
\hline $\mathrm{Ni} 2-\mathrm{P} 2-\mathrm{C} 71$ & $112.5(2)$ & $\mathrm{C} 16-\mathrm{C} 17-\mathrm{C} 18$ & $106(2)$ & $\mathrm{C} 45-\mathrm{C} 46-\mathrm{C} 47$ & $143(2)$ & $\mathrm{C} 74-\mathrm{C} 75-\mathrm{C} 76$ & $119.9(9)$ \\
\hline C59-P2-C65 & $101.4(3)$ & C17-C 18-C19 & $134(2)$ & $\mathrm{C} 46-\mathrm{C} 47-\mathrm{C} 48$ & $109(2)$ & C71-C76-C75 & $118.1(7)$ \\
\hline C59-P2-C71 & $104.4(3)$ & C18-C19-C20 & $115(2)$ & $\mathrm{C} 43-\mathrm{C} 48-\mathrm{C} 47$ & $114(1)$ & & \\
\hline C65-P2-C71 & $107.8(3)$ & C15-C20-C19 & $119(1)$ & O7-C49-08 & $125.0(7)$ & & \\
\hline
\end{tabular}

${ }^{a}$ Atoms marked with a prime are related to those at Table 3 by molecular symmetry centres.

bond; ${ }^{1,2}$ also the displacement at each $\mathrm{Ni}$ atom from the basal $\mathrm{O}_{4}$ plane is in a direction away from the other $\mathrm{Ni}$ atom in the dimers $[0.274(1) \AA$ for $\mathrm{Ni} 1$ and $0.277(1) \AA$ for Ni 2]. Average Ni-O bond lengths of 2.004(4) $\AA$ for 1a and $2.018(11) \AA$ for $\mathbf{1 b}$ fall within the range for such bonds found earlier. ${ }^{1-3}$ The apical Ni-P bond lengths are $\mathrm{Ni} 1-\mathrm{P} 1=2.386(2)$ and $\mathrm{Ni} 2-\mathrm{P} 2=2.389(2) \AA$, which are marginally longer than $2.368(1) \AA$ found for the corre-

Table 6. Least-squares planes in the molecules.

\begin{tabular}{|c|c|c|c|c|}
\hline $\begin{array}{l}\text { No. of } \\
\text { plane }\end{array}$ & Atoms in plane & $\Delta / \AA^{a}$ & $\begin{array}{l}\text { Distance to other } \\
\text { atoms } / \AA\end{array}$ & $\begin{array}{l}\text { Interplanar } \\
\text { angles }\end{array}$ \\
\hline 1 & $\mathrm{O} 1, \mathrm{O} 2^{\prime}, \mathrm{O} 3, \mathrm{O}^{\prime}$ & 0.003 & $\mathrm{Ni} 1 \quad 0.274$ & $1,2119.0(1)$ \\
\hline 2 & $05,06^{\prime}, 07,08^{\prime}$ & 0.001 & $\mathrm{Ni} 2 \quad 0.277$ & $3,4 \quad 89.7(1)$ \\
\hline 3 & $\mathrm{Ni} 1, \mathrm{O} 1, \mathrm{C} 1, \mathrm{O} 2, \mathrm{Ni}^{\prime}$ & 0.018 & P1 -0.082 & $5,6 \quad 88.0(1)$ \\
\hline 4 & $\mathrm{Ni} 1, \mathrm{O}, \mathrm{C} 11, \mathrm{O} 4, \mathrm{Ni}^{\prime}$ & 0.015 & 0.339 & \\
\hline 5 & $\mathrm{Ni} 2, \mathrm{O} 5, \mathrm{C} 39, \mathrm{O}, \mathrm{Ni2}$ & 0.037 & $P 2-0.419$ & \\
\hline 6 & $\mathrm{Ni} 2,07, \mathrm{C} 49,08, \mathrm{Ni}^{\prime}$ & 0.038 & $P 2-0.485$ & \\
\hline
\end{tabular}

${ }^{a}$ Max. deviation from plane. 


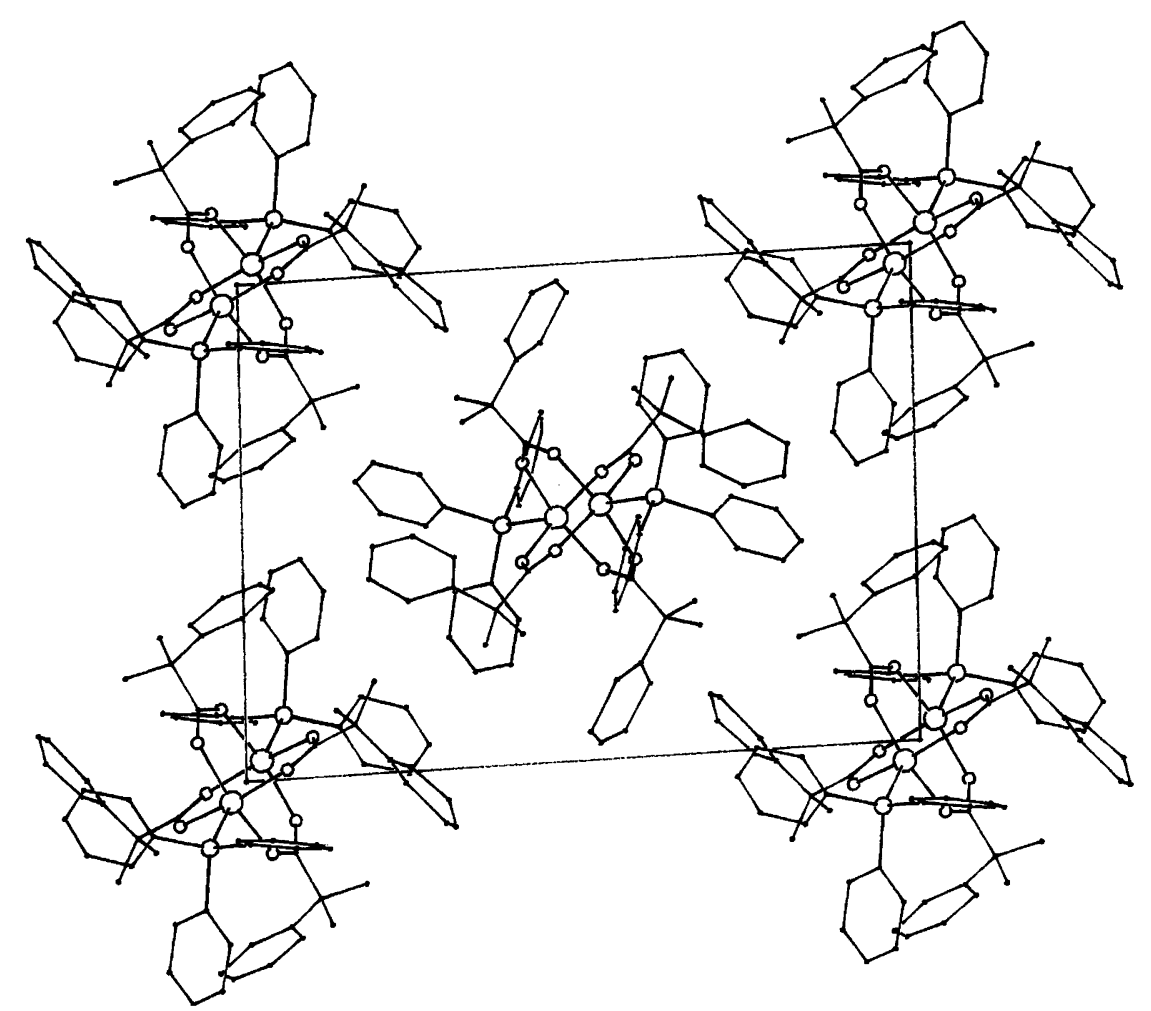

Fig. 2. The packing of the molecules in the unit cell as seen in the projection along the a-axis.

sponding bond length in $\left[\mathrm{Ni}\left(\mathrm{MePh} \mathrm{SiCOO}_{2} \mathrm{PPh}_{3}\right]_{2} \cdot{ }^{3}\right.$ The average dimensions of the bridging carboxyl groups in the dimers are $\mathrm{C}-\mathrm{O}=1.249(13) \AA 2, \mathrm{C}_{\mathrm{sp}^{2}} \mathrm{C}_{\mathrm{sp}^{3}}=$ 1.531(17) $\AA, \angle \mathrm{O}-\mathrm{C}-\mathrm{O}=124.7(9)^{\circ}$. Average total length of the $\mathrm{Ni}-\mathrm{O}-\mathrm{C}-\mathrm{O}-\mathrm{Ni}^{\prime}$ bridges is $6.545 \AA$.

Magnetic properties. The magnetic susceptibility data observed for the present compounds could not be fitted well to the Van Vleck equation, eqn. (3), for the coupling system of two $S=1$ spin states.

$$
\begin{aligned}
\chi_{\mathrm{A}}= & \frac{N g^{2} \beta^{2}}{k T}\left(\frac{5+\exp \left(\frac{-4 J}{k T}\right)}{5+3 \exp \left(\frac{-4 J}{k T}\right)+\exp \left(\frac{-6 J}{k T}\right)}\right) \\
& \times(1-P)+\frac{2}{3} \frac{N g_{\mathrm{im}}^{2} \beta^{2}}{k T} P+N \alpha
\end{aligned}
$$

However, as previously reported for the same type of $\mathrm{Ni}(\mathrm{II})$ dimers, a good fit for eqn. (4) was obtained for the magnetic data (Fig. 3). ${ }^{2}$

$$
\begin{aligned}
\chi_{\mathrm{A}}= & 2 \frac{N g^{2} \beta^{2}}{3 k T}\left[1+\frac{1}{3} \exp \left(\frac{-2 J}{k T}\right)\right]^{-1}(1-P) \\
& +\frac{2 N g_{\mathrm{im}}^{2} \beta^{2}}{3 k T} P+N \alpha
\end{aligned}
$$

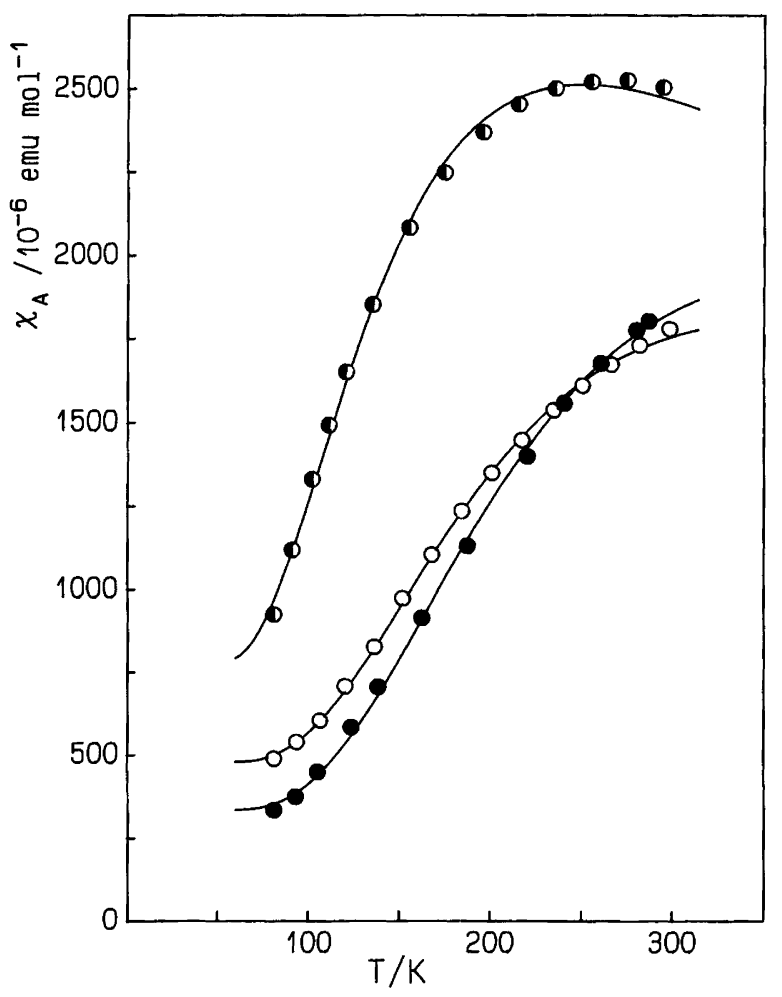

Fig. 3. Variation of magnetic susceptibility for some $\mathrm{Ni}(\mathrm{II})$ carboxylate dimers. D, $\left[\mathrm{Ni}\left(\mathrm{Me}_{2} \mathrm{PhCCOO}\right)_{2} \text { (quin) }\right]_{2} ; \mathrm{O}$, $\left[\mathrm{Ni}\left(\mathrm{Me}_{2} \mathrm{PhCCOO}\right)_{2} \mathrm{PPh}_{3}\right]_{2} ; \mathrm{O},\left[\mathrm{Ni}\left(\mathrm{Me}_{3} \mathrm{CCOO}\right)_{2}(2-\mathrm{pic})\right]_{2}$. 
The $g$-values in the previous report, however, were much larger than 2.2. The reason for this trend is apparent from the comparison between the $J=0$ extreme of eqns. (4) and (5):

$\chi_{\mathrm{A}}=\frac{N g^{2} \beta^{2} S(S+1)}{3 k T}=\frac{n(n+2)}{3} \frac{N g^{2} \beta^{2}}{4 k T}$

where $S$ is the total quantum number and $n$ is the number of unpaired electrons per metal ion. The expression indicates that the contribution of each electron to the magnetic susceptibility increases by $(n+2) / 3$ owing to intraatomic interaction of electronic spins. If the eqn. (4) type of treatment is possible, the general equation for a system with $n$ unpaired electrons per metal ion must be

$$
\begin{aligned}
\chi_{\mathrm{A}}= & \frac{(n+2)}{3} \sum_{i=1}^{n} \frac{N g_{i}^{2} \beta^{2}}{3 k T} \\
& \times\left[1+\frac{1}{3} \exp \left(\frac{-2 J_{i}}{k T}\right)\right]^{-1}+N \alpha
\end{aligned}
$$

When paramagnetic impurity is included in the expression. eqn. (6) is converted into eqn. (2). When $g$ is parametrized, a comparison of eqn. (2) with eqn. (4) gives the relation $g=(4 / 3)^{1 / 2} g_{i}$. For the application of eqn. (2), a single $g$-value for the electrons on the dimeric unit and a single $g_{\text {im }}$-value of the usual paramagnetic Ni(II) unit were used. This was done to avoid unreasonable convergence at the least-squares parameter fitting.

The $g$-values for $\left[\mathrm{Ni}\left(\mathrm{Me}_{3} \mathrm{CCOO}\right)_{2}(2.5-\mathrm{lu})\right]_{2},\left[\mathrm{Ni}\left(\mathrm{Me}_{2}-\right.\right.$ $\left.\mathrm{PhCCOO})_{2} \mathrm{PPh}_{3}\right]_{2}, \quad\left[\mathrm{Ni}\left(\mathrm{Me}_{3} \mathrm{CCOO}\right)_{2}(2.4-\mathrm{lu})\right]_{2}$, in the low- $T$ region $(T<200 \mathrm{~K})$, and $\left[\mathrm{Ni}\left(\mathrm{Me}_{2} \mathrm{PhCCOO}\right)_{2}-\right.$ (quin) $]_{2}{ }^{2,3}$ are $2.00,2.00,2.01$ and 2.03 , respectively. Those for $\left[\mathrm{Ni}\left(\mathrm{Me}_{3} \mathrm{CCOO}\right)_{2}(2 \text {-pic })\right]_{2},{ }^{3}\left[\mathrm{Ni}\left(\mathrm{Me}_{3} \mathrm{CCOO}\right)_{2}-\right.$ (2-Etpy) $]_{2}{ }^{2,3}$ and $\left[\mathrm{Ni}\left(\mathrm{Me}_{3} \mathrm{CCOO}\right)_{2}(2.4-\mathrm{lu})\right]_{2}{ }^{2}$ in the high- $T$ region $(T>200 \mathrm{~K})$ are $2.28,2.44$ and 2.71 , respectively. Thus, these compounds can be grouped by their $g$-values:

Group I for the compounds with $g$-values close to 2, and Group II for the compounds with larger $g$-values. The independent molecules of $\left[\mathrm{Ni}\left(\mathrm{Me}_{2} \mathrm{PhCCOO}\right)_{2} \mathrm{PPh}_{3}\right]_{2}$ which belong to Group I show no significant structural difference compared to each other or even to the structure of $\left[\mathrm{Ni}\left(\mathrm{Me}_{3} \mathrm{CCOO}\right)_{2}(2.4-\mathrm{lu})\right]_{2}$ (high- $T$ ) which belongs to Group II. The values of the exchange integrals, $-J$, were in the range of $126-224 \mathrm{~cm}^{-1}$, as shown in Table 2 . The $-J$-values are comparable to those determined by
Novotortsev et al. ${ }^{6}$ for $\left[\mathrm{Ni}\left(\mathrm{Me}_{3} \mathrm{CCOO}\right)_{2} \mathrm{~L}\right]_{2}(\mathrm{~L}=2$-pic, 4-pic, quin, and $\left.\mathrm{PPh}_{3} ;-J=150-157 \mathrm{~cm}^{-1}\right)$. Since Novotortsev et al. showed no $\chi_{\mathrm{A}}$ equation for the magnetic analysis, the magnetic property of $\left[\mathrm{Ni}\left(\mathrm{Me}_{3}-\right.\right.$ $\mathrm{CCOO})_{2}(2-$ pic $\left.)\right]_{2}$ was reinvestigated for comparison; the result is included in Table 2 . For the compounds listed in Table 2, no apparent correlation was found between their magnetic and structural properties. The only apparent difference in the two groups (I and II) is found in their molecular packing. At present, however, there are not enough data for further discussion. A wide range of magneto-crystal data are required to elucidate the mechanism of magnetic interaction in $\mathrm{Ni}(\mathrm{II})$ carboxylate dimers. In addition there is another problem to be solved. Since eqn. (4) is identical with the equation for spin equilibrium of the monomeric $\mathrm{Ni}$ (II) compound, a fit of $\chi-T$ data to eqn. (4) does not unequivocally lead to a spin-spin interaction between the two metal atoms in the dimeric unit. In order to reveal the non-existence of a monomeric spin equilibrium in this series of compounds, studies on $\mathrm{Ni}^{2+}$ $\mathrm{Zn}^{2+}$ heteronuclear carboxylate complexes are in progress. At present, although the purity of the $\mathrm{Ni}^{2+}$ $\mathrm{Zn}^{2+}$ dimer is not sufficient, the partial paramagnetic behaviour of the $\mathrm{Ni}^{2+}-\mathrm{Ni}^{2+}, \mathrm{Ni}^{2+}-\mathrm{Zn}^{2+}$, and $\mathrm{Zn}^{2}{ }_{-}^{-}$ $\mathrm{Zn}^{2+}$ mixtures suggests the non-existence of monomeric spin equilibrium on the $\mathrm{Ni}^{2+}$ ion in the $\mathrm{Ni}^{2+}-\mathrm{Zn}^{2+}$ dimer.

\section{References}

1. Kirillova, N. I., Struchkov, Yu. T., Porai-Koshits, M. A., Pasynskii, A. A., Antsyshkina, A. S., Minacheva, L. Kh., Sadikov, G. G., Idrisov, T. Ch. and Kalinnikov, V. T. Inorg. Chim. Acta 42 (1980) 115.

2. Hirashima, N., Husebye, S., Kato, M., Maartmann-Moe, K., Muto, Y., Nakashima, M. and Tokii, T. Acta Chem. Scand. 44 (1990) 984.

3. Morooka, M., Ohba, S., Nakashima, N., Tokii, T., Muto, Y., Kato, M. and Steward, O. Acta Crystallogr., Sect. C48 (1992) 1888.

4. Bencini, A., Benelli, C., Gatteschi, D. and Zanchini, C. $J$. Am. Chem. Soc. 102 (1980) 5820.

5. Uekusa, H., Ohba, S., Tokii, T., Muto, Y., Kato, M., Husebye, S., Steward, O. W., Chang, S.-C., Rose, J.P., Pletcher, J.F. and Suzuki, I. Acta Crystallogr., Sect. B48 (1992) 650.

6. Novotortsev, V. M., Rakitin, Yu. V., Pasynskii, A. A. and Kalinnikov, V. T. Dokl. Acad. Nauk. SSSR 240 (1978) 335.

7. Rakitin, T. V., Kalinnikov, V. T. and Eremin, M. V. Theor. Chim. Acta 45 (1977) 167.

Received December 27, 1993. 